\title{
Uma metodologia para parametrização do processo MIG/MAG CA
}

\author{
(A methodology for Parameterization of the AC MIG/MAG Process)
}

\author{
Américo Scotti ${ }^{1}$, Lindbergh Souza Monteiro ${ }^{2}$ \\ ${ }^{1}$ Centro para Pesquisa e Desenvolvimento de Processos de Soldagem (Laprosolda), Universidade Federal de Uberlândia, \\ Uberlândia, MG,Brasil (ascotti@ufu.br) \\ ${ }^{2}$ Instituto Federal de Educação Tecnológica do Maranhão (IFMA), São Luis, MA, Brasil (lindbergh.sm@gmail.com)
}

\begin{abstract}
Resumo
O processo MIG/MAG CA tem um potencial muito grande de aplicação, por permitir unir as características da soldagem MIG/MAG convencional (corrente contínua, com o eletrodo no positivo) com as de se usar corrente negativa na soldagem MIG/MAG. Entretanto, o formato de onda de corrente (alternada, pulsada no positivo e constante no negativo) demanda uma seleção criteriosa de seus inúmeros parâmetros de regulagem, o que vem limitando o estudo e aplicação desta versão de processo MIG/MAG. O objetivo deste trabalho foi propor e avaliar uma metodologia capaz de estimar os parâmetros de regulagem do processo MIG/MAG CA, de tal forma a se obter soldas com estabilidade de comprimento de arco e cordões com geometria adequada. É feita uma descrição passo a passo da definição dos parâmetros de entrada e da forma de se obter experimentalmente alguns valores de parâmetros necessários para estimação de outros valores de regulagem. As equações de estimação são apresentadas e discutidas. É feita uma demonstração da aplicação da metodologia, com a validação dos resultados pela comparação entre valores estimados e reais.
\end{abstract}

Palavras-chave: $M I G / M A G$, Corrente Alternada, polaridade negativa, seleção de parâmetros.

\begin{abstract}
The AC MIG/MAG process presents remarkable application potential, since it allows join the characteristics of the conventional MIG/MAG process (direct current, electrode positive) with the ones obtained when negative current is applied in MIG/ $M A G$ welding. However, the current wave shape (alternate, pulsed in positive and constant in negative polarities) demands a criterions selection of its innumerous setting parameters, fact that limits the development and application of this process version. The objective of this work was to propose and assess a methodology able to estimate the setting parameters of the CA MIG/MAG welding process, in such a way to result in welds with arc length stability and adequate bead geometry. A step-a-step description of the input parameter definitions and of the way to experimentally obtain some parameter values needed to estimate other setting parameters. The estimation equations are presented and discussed. A demonstration of the methodology application is carried out, with validation through actual welds.
\end{abstract}

Key-words: $M I G / M A G$, Alternate Current, negative polarity, parameter selection

\section{Introdução}

O processo de soldagem MIG/MAG é dos mais importantes processos de união de metais na atualidade. Isto se deve à sua alta capacidade de produção, característica constantemente cobiçada pelas indústrias em seus processos de fabricação. O Processo de soldagem MIG/MAG CA é uma variante do Processo $\mathrm{MIG} / \mathrm{MAG}$, no qual se procura combinar as vantagens típicas da soldagem MIG/MAG convencional (eletrodo atuando na polaridade Positiva, $\mathrm{CC}+$ ) com o aumento da taxa de deposição e redução do aporte térmico que ocorre quando o processo MIG/ MAG é operado com o Eletrodo na Polaridade Negativa (CC). Em $\mathrm{CC}+$, há ainda a possibilidade de soldar com diferentes modos de transferência metálica (curto-circuito, pulsado,

(Convidado em 15/05/2012; Texto final em 02/09/2012). goticular). Uma aplicação de destaque para este processo é a soldagem de chapas finas [1].

Um dos principais benefícios de se soldar em CC-seria o balanço reverso do calor produzido no arco. De acordo com Talkington [2], na soldagem MIG/MAG em CC+ usando como gás proteção uma mistura de Argônio e menos de $5 \%$ de $\mathrm{O}_{2}$ ou $\mathrm{CO}_{2}$, aproximadamente $30 \%$ do calor gerado no arco fica concentrado no eletrodo e o restante na peça (aproximadamente $70 \%$ ). Na soldagem emeste processo é contrário, cerca de $30 \%$ do calor gerado pelo arco é transferido para o metal de base e cerca de 70\% para o eletrodo. Souza et al. [3] e Farias et al [4] confirmaram que há uma maior taxa de fusão em CC-, mas os resultados indicam que esta se deva mais à escalada do arco pelas paredes da ponta do arame em procura por óxidos (para emissão por campo), aumentando a eficiência térmica, do que por um maior calor gerado na conexão catódica. Entretanto, Talkington [2] aponta que a soldagem em CC- é limitada, geralmente, ao tipo de transferência globular, sendo pouco usado na prática, por registrar grande instabilidade do arco e quantidade indesejável 


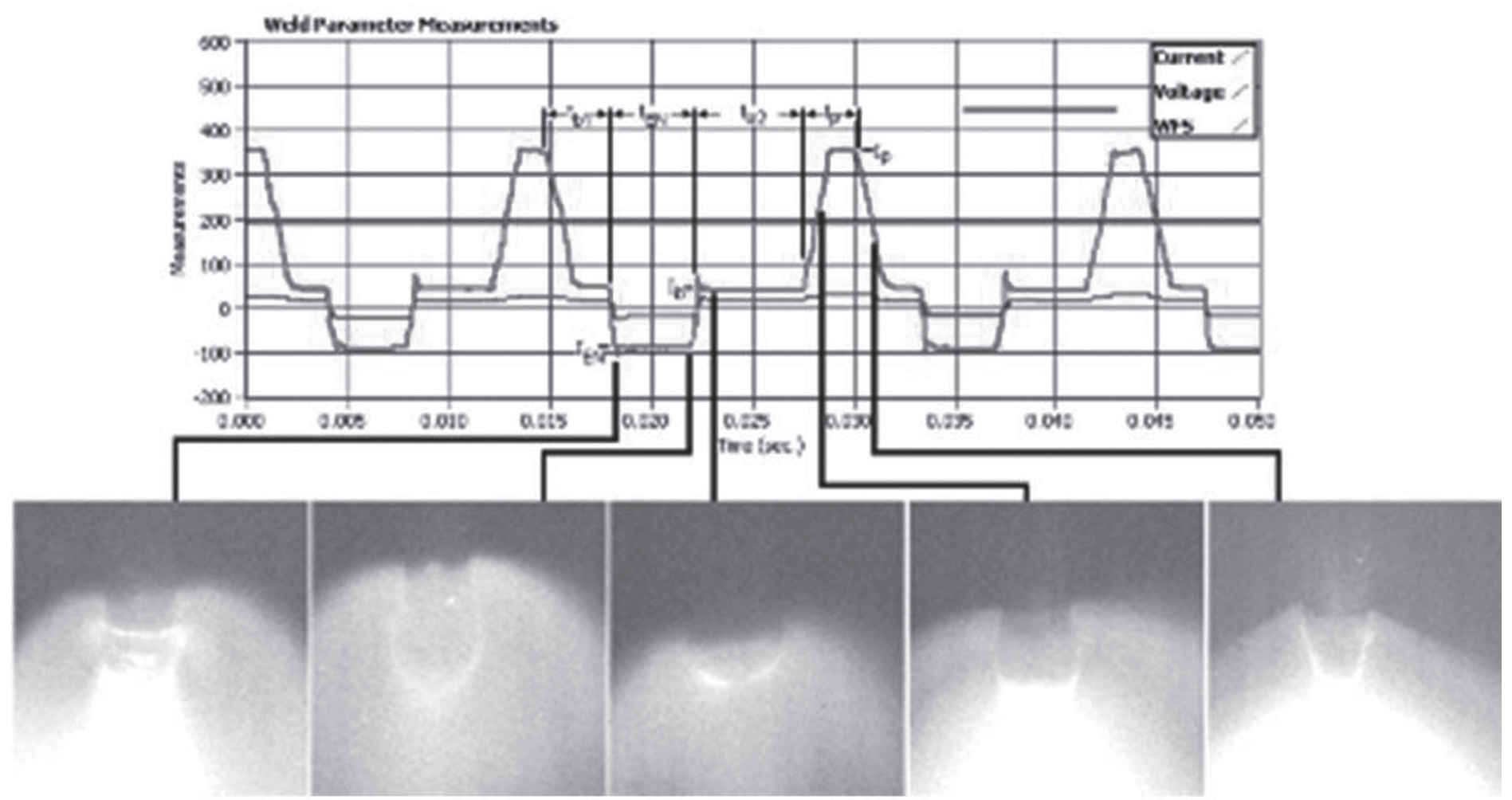

Figura 1. Formato típico de onda do processo MIG/MAG CA e a correlação com as fases de crescimento e destacamento da gota (adaptado de HARWIG [5])

de respingos produzidos. Estes resultados estão parcialmente em desacordo com Souza et al. [3], os quais demonstram que o modo de transferência metálica em CC- é dependente do tipo de gás de proteção utilizado e que é possível se obter transferência sem gotas repulsivas (globular e goticular) nesta polaridade.

Existem alguns tipos de ondas usadas nessa versão do processo $\mathrm{MIG} / \mathrm{MAG}$, sendo a pulsada $\mathrm{CC}+$ combinada com corrente constante CC-, representada na Figura 1, a mais aceita. Este formato de onda foi estudado por alguns autores, como Harwig et al [5] e Nascimento et al [6], para os quais o período inicia no polo negativo para formar a gota e após a reversão de corrente, existe um período de corrente de base no polo positivo (pré-pulso) para estabilizar a gota antes do pulso, etapa na qual a gota é lançada. Antes da nova reversão, após o destacamento da gota, existe mais um período de base no polo positivo.

Existem diversas outras formas de onda de corrente que podem ser utilizadas para o processo $\mathrm{MIG} / \mathrm{MAG} \mathrm{CA}$, não estando claro ainda, quais os benefícios de cada uma. Mas o que há de comum entre elas é o grande número de variáveis da onda a ser regulada. Alia-se a esta limitação a necessidade de se tentar manter o comprimento do arco o mais constante possível entre as inversões, fato que se consegue regulando a corrente no polo negativo para dar a mesma taxa de fusão do que no polo positivo. Assim, a mesma regulagem da velocidade de alimentação é usada nas duas polaridades. Como se vê, o número de parâmetros a regular é maior do que no MIG pulsado convencional (Quintino e Allum [7] e Vilarinho e Scotti [8]) e até de que o MIG/MAG duplo pulso [9]. Este fato é um fator desmotivante para o uso deste processo. Assim, o objetivo deste trabalho é apresentar uma metodologia para selecionar os parâmetros para soldagem com o processo MIG/MAG CA.

\section{Metodologia para Definir Parâmetros para Soldagem com MIG/MAG CA}

Neste processo, há uma transição periódica de polaridade, mas deve-se garantir que haja transferência metálica somente na polaridade positiva, através de um pulso. Assim, a estabilidade da transferência metálica está condicionada ao destacamento de uma única gota, com diâmetro próximo ao do arame-eletrodo, durante o tempo de pulso (UGPP). Além disso, deve-se procurar a manutenção de um mesmo comprimento do arco em ambas polaridades, para se garantir cordões regulares em dimensões e forma. Entretanto, na polaridade negativa há um aumento da taxa de fusão. Desta forma, para se manter uma mesma velocidade de alimentação em ambas as polaridades, uma opção é a diferenciação da corrente média para cada polaridade, a fim de se manter um mesmo comprimento de arco.

Foi, então, desenvolvida uma metodologia para encontrar parâmetros para soldagem em MIG CA que resultassem em boa estabilidade de transferência e em cordões com boa aparência e características geométricas dentro dos padrões de aceitabilidade. A aplicação dessa metodologia inicia-se com o formato de onda de corrente, por exemplo, como o da figura 1 (base-pulso-base). Em seguida devem ser definidos os parâmetros de entrada da regulagem para se soldar em MIG CA, ou sejam, os seguintes valores desejados: 
- A corrente média no polo positivo desejada $\left(\mathrm{I}_{\mathrm{m}+}\right)$;

- O valor de percentual de eletrodo no negativo (\%EN);

- O gás de Proteção;

- Composição e diâmetro do arame.

É importante ressaltar que não é possível ter como entrada diretamente a corrente média global desejada $\left(\mathrm{I}_{\mathrm{mg}}\right)$, isto por que ela é uma variável dependente de variáveis a determinar. Por isto, a entrada deve ser pela corrente média desejada na polaridade positiva $\left(\mathrm{I}_{\mathrm{m}}\right)$. Esta corrente vai ser próxima da corrente média final, mas, através de cálculos recursivos, podese chegar a corrente média global desejada. A corrente média na polaridade positiva a ser regulada deve ser típica para corrente média pulsada para a dada condição (material de base, tipo e diâmetro de eletrodo, gás de proteção e DBCP). Por exemplo, para o aço carbono, eletrodo AWS70S-6 com diâmetro de 1,2 mm e como gás de proteção uma mistura de $\mathrm{Ar}+2 \% \mathrm{O}_{2}$, os valores de corrente média devem variar entre 90 e $210 \mathrm{~A}$.

O percentual do eletrodo no negativo é descrito como a razão entre o tempo em que o eletrodo opera na polaridade negativa ( e o período. O período, por sua vez, corresponde ao tempo total despendido nas duas polaridades, ou seja: tempo no negativo ( mais o tempo no positivo (), conforme mostrado na equação 1 .

$\% E N=\frac{t_{E N}}{t_{E N}+t_{E P}}$

onde $\% E N=$ percentual do eletrodo no negativo, $=$ tempo que o eletrodo opera na polaridade negativa $\mathrm{e}=$ tempo que o eletrodo opera na polaridade positiva.

A escolha do tipo de gás de proteção depende do tipo de arame-eletrodo, que por sua vez depende do material a ser soldado. No caso da soldagem de aços estruturais, deve-se privilegiar um gás com baixo teor de $\mathrm{CO}_{2}$ ou de $\mathrm{O}_{2}$ em mistura com argônio, por facilitar a transferência metálica goticular durante os pulsos. Neste caso, o diâmetro do arame eletrodo mais usado é o de $1,2 \mathrm{~mm}$.

Como saída, espera-se um arco de comprimento em torno de $5 \mathrm{~mm}$, de forma constante, e a condição de Uma Gota Por Pulso (UGPP) na polaridade positiva, sem transferência metálica na polaridade negativa, com valores médios de corrente (Im), que poderá ser um pouco diferente do $\mathrm{Im}+$ estipulado, e de \%EN próximos dos valores desejados. Para se alcançar essas condições, algumas definições precisam ser tomadas, a saber:

a) Definição de valores para parâmetros que são invariáveis para uma dada condição:

- Corrente de pulso $\left(I_{p}\right)$;

- Corrente de base $1\left(I_{b 1}\right)$ e corrente de base $2\left(I_{b 2}\right)$;

- Distancia Bico de Contato Peça (DBCP);

- Comprimento livre do arame energizado $(L)$;

- As constantes da equação do consumo $\alpha$ e $\beta$ nas duas polaridades.
A corrente de pulso $\left(I_{p}\right)$ é definida de acordo com a sua aplicação. Se o objetivo for maior penetração, deve-se adotar o seu valor muito maior que a corrente de transição estabelecido em tabelas ou determinar experimentalmente para o gás e arame escolhidos, ou seja: $\left(\mathrm{I}_{\mathrm{p}}\right)>>\left(\mathrm{I}_{\text {transição }}\right)$. No caso de se querer fazer revestimento, deve-se escolher um valor de $\left(\mathrm{I}_{\mathrm{p}}\right)$ um pouco maior do que $\left(\mathrm{I}_{\text {transição }}\right)$. A corrente de base $\left(\mathrm{I}_{\mathrm{b}}\right)$, na pratica $\left(\mathrm{I}_{\mathrm{b} 1}\right)$ ou $\left(\mathrm{I}_{\mathrm{b} 2}\right)$, deve ser escolhida também de acordo a corrente média desejada. O valor da corrente de base recomendada deve estar acima de 40 e 60 A. Para a DBCP, procura-se privilegiar o que normalmente é praticado para o MIG Pulsado; deve-se tentar o maior valor para se conseguir alta taxa de alimentação usando valores baixos de corrente média (abaixo da corrente de transição), aceitandose um limite de $25 \mathrm{~mm}$ para arame-eletrodo de $1,2 \mathrm{~mm}$ de diâmetro, por questões de rigidez da extremidade livre do arame. O comprimento livre do eletrodo é normamente a DBCP subtraída do comprimento do arco, que na pratica adota-se para este caso $5 \mathrm{~mm}$. Finalmente, as constantes da equação geral do consumo, são determinadas experimentalmente para o conjunto gás-arame-polaridade. Caso o usuário não as tenha tabelado, deve procurar determiná-las ou solicitar a um laboratório especializado esta determinação, conforme procedimentos descritos no item 2.1.

b) Definição pelo usuário de valores para demais parâmetros:

- Tempo de pulso $\left(\mathrm{t}_{\mathrm{p}}\right)$;

- Tempo de base $\left(\mathrm{t}_{\mathrm{b}}\right)$;

- Corrente RMS na polaridade positiva $\left(\mathrm{I}_{\mathrm{RMS}}\right)$;

- Corrente no negativo $\left(\mathrm{I}_{\mathrm{n}}\right)$;

- Tempo no negativo $\left(\mathrm{t}_{\mathrm{n}}\right)$;

- Corrente média global

O tempo de pulso $\left(t_{p}\right)$ é calculado pela curva de interpolação $\mathrm{Im}+\mathrm{xt}_{\mathrm{p}}$ para a condição de UGPP, levantada experimentalmente para as condições em MIG Pulsado (caso o usuário não tenha esta curva, deve procurar determiná-la ou solicitar a um laboratório especializado esta determinação, conforme procedimentos descritos no item 2.2). O tempo de base ( $\mathrm{t}_{\mathrm{b}}$ ) é calculado pela equação da corrente média exponencial para MIG Pulsado, ou seja, isolando-se o tempo de base na equação 2 e entrando-se com os valores dos demais parâmetros, onde $\mathrm{Im}+$ é um valor de entrada e são as constantes exponenciais de tempo do formato de onda exponencial (Figura 2), escolhidos devido às características da fonte utilizada. Para tal, é utilizado um programa usando-se a ferramenta MATLAB para encontrar a solução da equação.

$I_{m+}=\frac{I_{p} \cdot t_{p}-\frac{\left(I_{p}-I_{b}\right)}{\lambda_{1}}\left(1-\mathrm{e}^{\lambda_{1} \cdot t_{p}}\right)+I_{b} \cdot t_{b-\frac{\left(I_{b}-I_{p}\right)}{\lambda_{2}}\left(1-\mathrm{e}^{\left.-\lambda_{2} \cdot t_{b}\right)}\right.}}{t_{p}+t_{b}}$ 


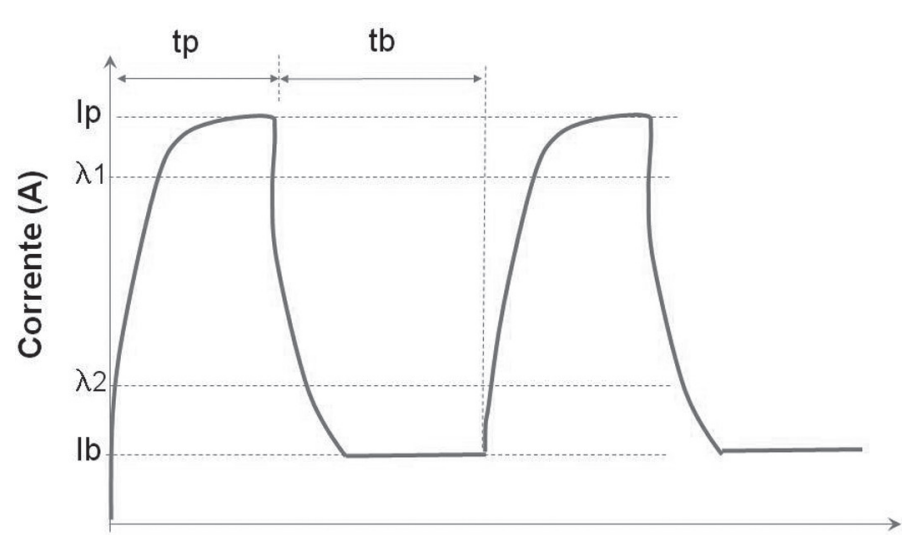

\section{Tempo (ms)}

Figura 2. Formato de onda para corrente pulsada do tipo Exponencial, típica de equipamentos com capacidade de corrente pulsada

A corrente RMS ( $\left.I_{R M S}\right)$ é calculada pela equação 3, a partir do formato de onda exponencial, na condição de UGPP;

$$
\begin{aligned}
& I_{R M S}=\left\{I_{p}^{2} \cdot t_{p}+I_{b}^{2} \cdot t_{b}-\frac{\left(I_{p}-I_{b}\right.}{\mathrm{e}^{\lambda_{1} t_{p}} \cdot \lambda_{1}}\left(\frac{\left(I_{p}-I_{b)}\right.}{2 \mathrm{e}^{\lambda_{1} \cdot t_{p} \cdot \lambda_{1}}}+2 \cdot I_{p}\right)\right. \\
& -\frac{\left(I_{b}-I_{p)}\right.}{\mathrm{e}^{\lambda_{2} \cdot t_{p}} \lambda_{2}}\left(\frac{\left(I_{b}-I_{p}\right)}{2 \mathrm{e}^{\lambda_{2} \cdot t_{b} \cdot \lambda_{1}}}-2 I_{b}\right) \\
& -\frac{\left(I_{p}-I_{b}\right)}{\mathrm{e}^{\lambda_{1} \cdot t_{p} \cdot \lambda_{1}}}\left(3 \cdot I_{p}+I_{b}-\frac{\left(I_{b}-I_{p}\right)}{2 \cdot \lambda_{2}}\left(3 \cdot I_{p}+I_{p}\right\}\right.
\end{aligned}
$$

A corrente no negativo $\left(I_{n}\right)$ é calculada igualando-se os valores da taxa de fusão nas duas polaridades, expressas pelas equações 4 e 5 .

$V_{\text {alim }}{ }^{+}=\alpha^{+} I_{m+}+\beta^{+} L I_{R M S+}^{2}$

$V_{\text {alim }}{ }^{-}=\alpha^{-} I_{m-}+\beta^{-} L I_{R M S-}^{2}$

onde é um parâmetro de entrada (corrente média desejada no polo positivo), $\mathrm{I}_{\mathrm{RMS}}+$ calculado de acordo com equação 3 , $I_{\text {RMS- }}$ é por definição $\left(I_{n}\right)$, uma vez que no negativo a corrente é constante, e culados experimentalmente.

Igualando-se o consumo nas duas polaridades, tem-se:

$V_{\text {alim }}{ }^{-}-V_{\text {alim }}{ }^{+}=0$

Substituindo-se a equação 4 e 5 em 6, tem-se:

$\alpha^{-} I_{n}+\beta^{-} L I_{n}^{2}-V_{\text {alim }}{ }^{+}=0$

que reordenada apresenta-se na forma da equação 8

$\beta^{-} L I_{n}^{2}+\alpha^{-} I_{n}-V_{\text {alim }}{ }^{+}=0$

Assim, o valor da corrente no negativo é calculado pela solução da equação 8. Já o valor do tempo no negativo é calculado a partir da equação 9:

$t_{n}=\frac{\% E N t_{p p}}{1-\% E N}$

onde $t_{n}=$ tempo em que o eletrodo opera na polaridade negativa $\mathrm{e}=$ tempo total na polaridade positiva.

Finalmente, a corrente média global $\left(\mathrm{I}_{\mathrm{mg}}\right)$, que corresponde à média ponderada das correntes nas diversas fases do pulso, é definida pela equação 10 ;

$I_{m g}=\frac{I_{p} t_{p}+I_{b} t_{b}+\left|I_{n}\right| t_{n}}{t_{p}+t_{b}+t_{n}}$

Onde,

$\mathrm{I}_{\mathrm{p}}=$ Corrente de pulso $\left(\mathrm{I}_{\mathrm{p}}\right)$;

$\mathrm{I}_{\mathrm{b}}=$ Corrente de base; $\left(\mathrm{I}_{\mathrm{b} 1}\right)$ ou $\left(\mathrm{I}_{\mathrm{b} 2}\right)$;

$\mathrm{t}_{\mathrm{p}}=$ tempo de pulso;

$\mathrm{t}_{\mathrm{b}}=$ tempo de base;

$\mathrm{I}_{\mathrm{n}}=$ corrente no negativo;

$t_{n}=$ tempo no negativo

No caso da corrente média global $\left(I_{\mathrm{mg}}\right)$ não alcançar após os cálculos o valor desejado, de forma recursiva pode-se aumentar ou diminuir a corrente média no polo positivo $(\mathrm{Im}+)$ e reiniciar os cálculos até a convergência do valor de $\mathrm{I}_{\mathrm{mg}}$. ao desejado.

\subsection{Determinação experimental das constantes da equação do consumo na soldagem em polaridade negativa e em polaridade positiva $(\mathrm{CC}+)$}

O consumo nas polaridades positiva e negativa foram expressos pelas Equações 4 e 5, respectivamente. Para determinar as constantes $\alpha+$ e $\beta+, \alpha$ - e $\beta$ - dessas equações, uma série de experimentos tem de ser feita, varrendo-se uma faixa de corrente aplicável para o dado arame e gás de proteção (a rigor, esta varredura também deveria se dar para a distância bico de contato-peça, mas pode-se assumir um valor médio de $20 \mathrm{~mm}$ como representativo para a faixa a ser utilizada nesta técnica, a saber, de 15 a $25 \mathrm{~mm}$ ). Durante as soldagens, tentar manter o arco em torno de $5 \mathrm{~mm}$, comprimento mais utilizado para este tipo de processo. Isto é possível caso o equipamento tenha o controle adaptativo de comprimento do arco, ajustando-se a tensão de referência até que se alcance o comprimento de arco desejado. Caso não tenha, a velocidade de alimentação deve ser ajustada para mais e para menos até que se alcance o valor desejado.

Para o caso da polaridade positiva, deve-se regular a fonte de soldagem no modo corrente constante, com pulsação. Caso a fonte tenha um controle sinérgico para corrente pulsada, usá-lo para cada corrente média desejada, em função do gás de proteção e arame escolhidos. Caso contrário, deve-se regular todos os parâmetros de pulsação, tomando-se como base os valores de correntes de pulso e tempo de pulso que serão utilizados no trabalho. De qualquer forma, é importante garantir a condição de destacamento de uma gota por pulso para cada nível de corrente desejada, por exemplo, utilizando-se de monitores ópticos [10] ou filmagem de alta velocidade [11]. Para o caso da polaridade negativa, deve-se regular a fonte de soldagem no modo corrente constante, sem pulsação (caso a fonte não permita, colocar no 
modo pulsado, mas fazendo $\left.\mathrm{I}_{\mathrm{p}}=\mathrm{I}_{\mathrm{b}}\right)$.

Durante as soldagens, monitorar a velocidade de alimentação (Valim+ ou Valim-) e a corrente e determinar para cada experimento os valores de $\mathrm{I}_{\mathrm{m}^{+}}$e $\mathrm{I}_{\mathrm{rms}^{+}}$ou $\mathrm{I}_{\mathrm{m}-}$ e $\mathrm{I}_{\mathrm{rms}-}$. Assim, através de análise de regressão, disponível em programas computacionais comerciais, é possível determinas as constantes, tomando como base a Equação 4 ou a 5 .

\subsection{Determinação experimental dos tempos de pulso $\left(t_{p}\right) \mathrm{em}$ polaridade positiva $(\mathrm{CC}+)$}

O procedimento experimental para determinar o tempo de pulso $\left(\mathrm{t}_{\mathrm{p}}\right)$ para uma dada corrente de pulso $\left(\mathrm{I}_{\mathrm{p}}\right)$ consiste em fazer vários cordões de solda, variando-se escalonadamente o tempo de pulso $\left(\mathrm{t}_{\mathrm{p}}\right)$ para cada corrente média para qual esse parâmetro será regulado (varrer a corrente média dentro da faixa de aplicabilidade). A velocidade de alimentação (Valim) deve ser condizente com cada corrente média, procurando-se um arco de aproximadamente $5 \mathrm{~mm}$ (iniciava-se a soldagem com um valor alto da Valim e abaixa-se progressivamente essa regulagem até se conseguir abrir o arco e mantê-lo no comprimento desejado). $\mathrm{O}$ valor de $\mathrm{I}_{\mathrm{b}}$ deve ser previamente escolhido, de forma similar ao Ip. Assim, para cada regulagem do tempo de pulso $\left(\mathrm{t}_{\mathrm{p}}\right)$, será necessário ajustar o tempo de base $\left(\mathrm{t}_{\mathrm{b}}\right)$, mantendo-se a corrente média, como indicado pela equação 11. Também haverá necessidade de se ajustar o comprimento do arco, através da velocidade de alimentação.

$t_{b}=\frac{t_{p}\left(I_{m}+I_{p}\right)}{I_{b}+I_{m}}$

Existirá uma faixa de tempos de pulso em que se alcançará a condição de uma gota por pulso para cada nível de corrente média desejada, para uma dada corrente pulso-arame-gás de proteção. Para medir se estaria havendo a condição de uma gota por pulso, pode-se usar, por exemplo, monitores ópticos [10] ou filmagem de alta velocidade [11]. Assim, a partir dos valores regulados e dos valores monitorados é construído um gráfico da relação corrente média e tempo de pulso médio da faixa em que se tem a condição de uma gota por pulso, como ilustra a Figura 3.

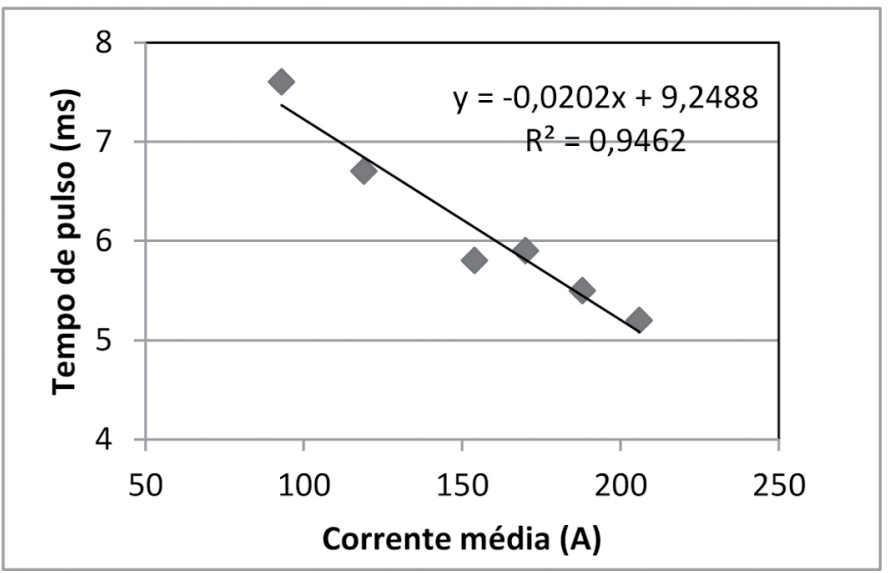

Figura 3. Relação entre corrente média e tempo de pulso levantada para um $I_{p}=250 \mathrm{~A}$, arame AWS ER70S-6 e proteção com usando uma mistura de $\mathrm{Ar}+2 \% \mathrm{O} 2$

\section{Aplicação da Metodologia}

Para exemplificar a aplicação da metodologia descrita, o metal de base utilizado foi o aço estrutural ao carbono, sem liga. Assim, como material de deposição foi especificado o eletrodo da classe AWS ER70S-6, com diâmetro de 1,2 mm, mantendose uma DBCP de $25 \mathrm{~mm}$. Uma mistura de Argônio $+2 \%$ de Oxigênio foi usada, visando uma condição de $50 \% \mathrm{EN}$.

Inicialmente foram fixados os seguintes valores dos parâmetros de entrada de corrente média na polaridade positiva $(\operatorname{Im}+)=90 \mathrm{~A} ; 120 \mathrm{~A} ; 150 \mathrm{~A} ; 165 \mathrm{~A} ; 180 \mathrm{~A}$ e 210 A. Foram também definidos os outros parâmetros de entrada que são invariáveis, ou seja:

- $\mathrm{I}_{\mathrm{p}}=250 \mathrm{~A}$;

- Corrente de base $1\left(\mathrm{I}_{\mathrm{b} 1}\right)$ ou corrente de base $2\left(\mathrm{I}_{\mathrm{b} 2}\right)=40 \mathrm{~A}$;

- $\mathrm{DBCP}=25 \mathrm{~mm}$, e uma $\mathrm{L}_{\text {arco }}=5 \mathrm{~mm}$;

- Valores das constantes da equação do consumo nas duas polaridades (levantadas experimentalmente):

○ Na polaridade negativa:

$$
\alpha^{-}=6,19 \times 10^{-4} \mathrm{~m} / \mathrm{s} . \mathrm{A} ; \beta^{-}=2,9 \times 10^{-5} \mathrm{~s}^{-1} \mathrm{~A}^{-2} ;
$$

- Na polaridade positiva:

$$
\alpha^{+}=1,19 \times 10^{-4} \mathrm{~m} / \mathrm{s} . A ; \beta^{+}=8,9 \times 10^{-5} s^{-1} A^{-2} ;
$$

Em seguida foi definido o conjunto dos parâmetros estimados, a saber:

- Os valores de tempo de pulso $\left(\mathrm{t}_{\mathrm{p}}\right)$, calculados pela curva de interpolação $\mathrm{I}_{\mathrm{m}+} \times \mathrm{t}_{\mathrm{p}}$ levantada experimentalmente para todas as correntes médias no polo positivo, já definidos;

- O tempo de base $\left(\mathrm{t}_{\mathrm{b}}\right)$; isolando-se o tempo de base na equação 2 e entrando-se com os valores dos demais parâmetros $\left(I_{m+}, I_{p}, I_{b}, t_{p}, \lambda_{1}\right.$ e $\lambda_{2}$, esses últimos assumidos como 2 e 3 respectivamente, em função do equipamento de soldagem);

- Os valores de velocidade de alimentação na polaridade positiva, para cada valor de corrente média de entrada, calculados pela equação 4 ;

- Os valores de velocidade de alimentação na polaridade negativa, para cada valor de corrente média de entrada, calculado pela equação 5;

- Os valores de corrente no negativo $\left(\mathrm{I}_{\mathrm{n}}\right)$, para cada valor de corrente média de entrada, calculado utilizando-se a equação 8 ;

- Os valores do tempo no negativo $\left(\mathrm{t}_{\mathrm{n}}\right)$, calculados fazendo uso da equação 9;

- Finalmente, os valores de corrente média global $\left(\mathrm{I}_{\mathrm{mg}}\right)$, através da equação 10 .

A Tabela 1 expressa os valores de entrada e estimados pela metodologia proposta para a faixa de corrente de 90 a 210 A e um $\mathrm{EN}=50 \%$. Pode-se perceber que a corrente média global foi uma pouco menor do que a corrente média no polo positivo. Assim, caso fosse desejo um aumento deste valor, seria apenas 
Tabela 1. Parâmetros estimados para soldagem MIG/MAG CA, para uma faixa de corrente no eletrodo positivo de 90 a 210 A e EM= $50 \%$

\begin{tabular}{|c|c|c|c|c|c|c|c|c|c|c|c|c|}
\hline \multicolumn{9}{|c|}{ Parâmetros de Entrada } & \multicolumn{9}{|c|}{ Parâmetros Estimados } \\
\hline $\begin{array}{c}I_{m}^{+} \\
(A)\end{array}$ & $\begin{array}{c}E M \\
\%\end{array}$ & $\begin{array}{c}I_{p} \\
(A)\end{array}$ & $\begin{array}{c}I_{b 1} \\
(A)\end{array}$ & $\begin{array}{c}I_{b 2} \\
(A)\end{array}$ & $\begin{array}{c}t_{p} \\
(m s)\end{array}$ & $\begin{array}{c}t_{b 1} \\
(m s)\end{array}$ & $\begin{array}{c}t_{b 2} \\
(m s)\end{array}$ & $\begin{array}{c}V_{\text {alim }} \\
(\mathrm{m} / \mathrm{min})\end{array}$ & $\begin{array}{c}I_{n} \\
(A)\end{array}$ & $\begin{array}{c}t_{n} \\
(m s)\end{array}$ & $\begin{array}{c}I_{m g} \\
(A)\end{array}$ & $\begin{array}{c}T \\
(m s)\end{array}$ \\
\hline 90 & 0,5 & 250 & 40 & 40 & 7,4 & 22,30 & 1 & 2,5 & 63 & 30,7 & 77 & 61,5 \\
\hline 120 & 0,5 & 250 & 40 & 40 & 6,8 & 9,60 & 1 & 3,4 & 80 & 17,4 & 101 & 34,8 \\
\hline 150 & 0,5 & 250 & 40 & 40 & 6,2 & 4,23 & 1 & 4,7 & 100 & 11,4 & 127 & 22,8 \\
\hline 165 & 0,5 & 250 & 40 & 40 & 5,9 & 2,73 & 1 & 5,2 & 126 & 9,6 & 147 & 19,2 \\
\hline 180 & 0,5 & 250 & 40 & 40 & 5,6 & 1,55 & 1 & 6,1 & 134 & 8,1 & 159 & 16,2 \\
\hline 210 & 0,5 & 250 & 40 & 40 & 4,9 & $-0,05$ & 1 & 6,7 & 166 & 5,9 & 191 & 11,8 \\
\hline
\end{tabular}

$I_{m}{ }^{+}=$Corrente média na polaridade positiva; $\% E N=$ Percentual de eletrodo no negativo; $I_{p}=$ corrente de pulso; $I_{b 1}=$ corrente de base antes do pulso; $I_{b 2}=$ corrente de base após o pulso; $t_{p}=$ tempo de pulso; $t_{b 1}=$ Tempo de base antes do pulso; $t_{b 2}=$ tempo de base após o pulso; $V_{a}=$ Velocidade de alimentação; $I_{n}=$ Corrente no negativo; $t_{n}=$ tempo no negativo;

$I\left(_{\text {ma }}=\right.$ Corrente média absoluta; $T=$ Período.

Tabela 2. Comparação entre os valores de Corrente Média Global Estimada e Corrente Média Global Monitorada, com o respectivo erro percentual, para soldagens realizadas em soldagem MIG/MAG CA usando como parâmetros de regulagem os valores de entrada e estimados mostrados na Tabela 2

\begin{tabular}{|c|c|c|}
\hline $\begin{array}{c}\mathrm{I}_{\mathrm{mg}} \\
\text { (Estimado) } \\
(\mathrm{A})\end{array}$ & $\begin{array}{c}\mathrm{I}_{\mathrm{mg}} \\
\text { (Medido) } \\
(\mathrm{A})\end{array}$ & $\begin{array}{c}\text { Erro Percentual } \\
(\%)\end{array}$ \\
\hline 78 & 76 & 2,7 \\
\hline 99 & 101 & $-2,2$ \\
\hline 125 & 122 & 2,7 \\
\hline 146 & 144 & 1,1 \\
\hline 161 & 156 & 3,4 \\
\hline 186 & 187 & $-0,7$ \\
\hline
\end{tabular}

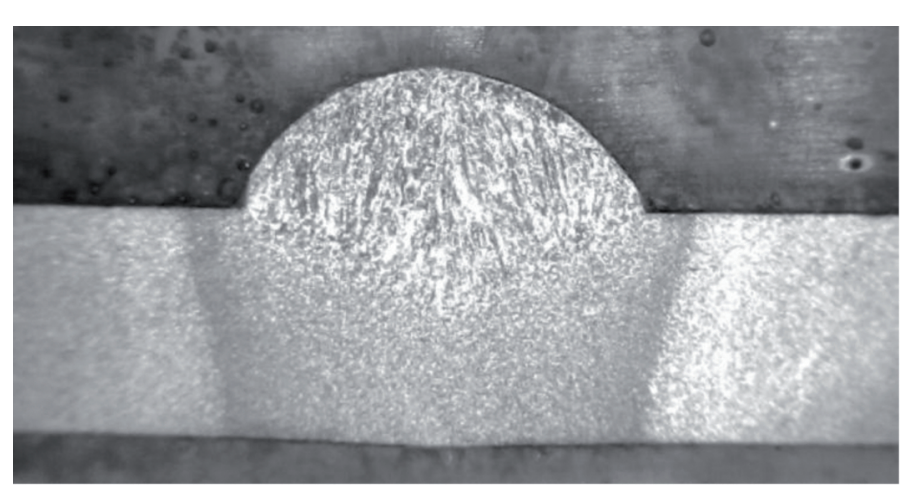

Figura 4. Seção transversal de um cordão de solda de simples deposição sobre chapa usando o $\mathrm{MIG} / \mathrm{MAG} \mathrm{CA}(\mathrm{EN}=50 \%$; $I_{p}=250 A ; I_{b}=40 A ; I_{n}=126 A ; t_{p}=5,9 m s ; t_{b 1}=2,7 m s ; t_{b 2}=1$ $\left.\mathrm{ms} ; \mathrm{t}_{\mathrm{EN}}=9,6 \mathrm{~ms}\right)$

necessário por tentativa e erro corrigir o valor de entrada para a corrente média no polo positivo $\left(\mathrm{I}_{\mathrm{m}+}\right)$. Usando-se os valores de entrada $\left(I_{p}, I_{b 1}\right.$ e $\left.I_{b 2}\right)$ e os valores estimados $\left(t_{p}, t_{b 1}, t_{b 2}, V_{a l i m}, I_{n} e\right.$ $\mathrm{t}_{\mathrm{n}}$ ) da Tabela 1 para regular os equipamentos, soldagens foram feitas para validar a metodologia. A Tabela 2 mostra o parâmetro de validação, no caso a corrente média global $\left(\mathrm{I}_{\mathrm{mg}}\right)$, uma vez que este foi estimado e pode ser monitorado. Pode-se perceber o bom desempenho da metodologia.

A Figura 4 ilustra a seção transversal de um cordão sobre chapa realizado com o processo $\mathrm{MIG} / \mathrm{MAG} \mathrm{CA}$ e regulagem dos parâmetros com valores estimados pela metodologia proposta, evidenciando a qualidade geométrica do cordão produzido.

\section{Conclusões}

Com a metodologia proposta é possível calcular com grande precisão os valores a serem usados na regulagem de um equipamento para soldagem com $\mathrm{MIG} / \mathrm{MAG} \mathrm{CA}$, superando uma das limitações desta versão do processo MIG/MAG. Apesar de demandar levantamento experimental de alguns parâmetros, a confecção de um banco de dados ao longo do tempo vai tornando mais fácil e popular a aplicação do processo pela maior facilidade de seleção dos seu parâmetros de regulagem. 


\section{Agradecimentos}

Os autores deste trabalho gostariam de agradecer à CAPES, através do Programa Dinter IFMA-UFU, pela oportunidade para um dos autores desenvolver seu projeto de pesquisa no Grupo Centro para Pesquisa e Desenvolvimento de Processos de soldagem da UFU. Também agradecem ao CNPq, através do processo 302091/2011-4, pela bolsa de pesquisa de um dos autores, e à Fapemig, através do projeto RDP-00140-10, pelo suporte financeiro laboratorial.

\section{Referências Bibliográficas}

[1] UEYAMA, T., TONG, H., HARADA, S., PASSMORE, R., e USHIO M., AC Pulsed GMAW Improves Sheet Metal Joining, Welding Journal, 84 (2), 2005, pp. 40-46.

[2] TALKINGTON, J.E., Variable polarity gas metal arc welding. MSc Thesis, The Ohio State University. 1998. 113p.

[3] SOUZA, D., RESENDE, A.A., SCOTTI, A., Um Modelo Qualitativo para Explicar a Influência da Polaridade na Taxa de Fusão no Processo MIG/MAG, Soldagem \& Inspeção, 14 (3), 2009, pp. 192-198.

[4] FARIAS, J.P., MIRANDA, H.C., MOTTA, M.F., PAIVA, F.D.Q., PESSOA, E.F., Efeito da Soldagem MIG/MAG em Corrente Alternada sobre a Geometria da Solda, Soldagem \& Inspeção, 10 (4), 2005, pp. 173-181.

[5] HARWIG, D.D., DIERKSHEIDE, J.E., YAPP, D., DLCKMAN, S., Droplet burnoff rate measurements on the VPGMAW process, Welding journal, 8(3), 2006, pp. 52s-62s (ISSN 0043-2296).

[6] NASCIMENTO, A.S., FERNANDES, D.B., MOTA, C.A.M., VILARINHO, L.O., Metodologia para Determinação de Parâmetros para Soldagem MIG com Polaridade Variável, Soldagem \& Inspeção, 13 (2), 2008, pp. 97-104

[7] QUINTINO, M.L.; ALLUM, C.J. Pulsed GMAW Interactions and Process Parameters Parts 1 \& 2. Weld. \& Metal Fab.,52 (2 and 4), 1984.

[8] VILARINHO, L. O. \& SCOTTI, A., An Alternative Algorithm for Synergic Pulsed GMAW of Aluminum, In: Australasian Welding Journal, vol 45, 2nd Quarter, 2000, pp. 3644 (ISSN1039-0642) (http://www.hera.org.nz/Awj/Awj.html).

[9] SILVA, C.L.M., \& SCOTTI, A, The Influence of Double Pulse on Porosity Formation in Aluminum GMAW, Journal of Materials Processing Technology, Elsevier Science Publ, Holland, 171 (3), 2006, pp. 366-372. (ISSN: 0924-0136) doi:10.1016/j.jmatprotec.2005.07.008.

[10] MIRANDA, H.C.; SCOTTI, A.; FERRARESI, V.A., Identification and control of metal transfer in pulsed GMAW using optical sensor, Science and Technology of Welding \& Joining, Maney Publishing, Volume 12, Number 3, May 2007, pp. 249-257 (DOI: 10.1179/174329307X164229) (ISSN 13621718, Online ISSN: 1743-2936) .

[11] BÁLSAMO, P.S.S., VILARINHO, L. O., VILELA, M. \& SCOTTI, A.; Development of an Experimental Technique for Studying Metal Transfer in Welding: Synchronized Shadowgraphy, In: Int. J. for the Joining of Materials, vol 12, no. 1, 2000, The European Institute for Joining of Materials (JOM),
Denmark, pp. 1-12 (ISSN 0905-6866). 